\title{
Lunar Reconnaissance Orbiter (LRO) Thermal On-Orbit Performance
}

\author{
Charles Baker ${ }^{1}$ \\ NASA/Goddard Spaceflight Center, Greenbelt, MD, 20771 \\ and \\ Christine Cottingham ${ }^{2}$ and Sharon Peabody ${ }^{3}$ \\ Edge Space Systems, 13860 Kennard Drive, Glenelg, MD 21737
}

\begin{abstract}
The Lunar Reconnaissance Orbiter (LRO) was launched on June 18, 2009. The nominal mission ended on September 15, 2010 and LRO is now on a four-year extended mission. The LRO performances in hot and cold cases are compared to pre-launch analysis predicts, and operational lessons learned are discussed. One instrument has required tighter-thananticipated thermal control, and two others have frequently requested unanticipated calibration maneuvers that had to be evaluated for their thermal performance. A series of off nadir thermal analyses of the entire orbiter were performed prior to launch, and these predictions are compared to actual maneuvers, with a discussion of the process by which maneuvers can be rapidly evaluated for thermal concerns. On December $21^{\text {st }}$, 2010, LRO experienced its first severe Lunar Eclipse. Operationally, this required the Spacecraft to pre-heat its main avionics panel in order to minimize control heater power during the period when the Earth blocks the sun from the moon. The operational design and in-flight performance are summarized.
\end{abstract}

\section{Nomenclature}

$q_{I R}^{\prime \prime} \quad=$ Infared flux from Lunar Surface

$C_{1} \quad=$ Peak flux at subsolar point

$C_{2}=$ Minimum flux emitted from shaded Lunar surface

$\beta \quad=$ Beta Angle

$\theta \quad=$ Orbit Angle from Subsolar Point (Dayside Only)

\section{Introduction}

The Lunar Reconnaissance Orbiter (LRO) was launched on June 18, 2009. The nominal mission ended on September 15, 2010 and LRO is on a four year extended mission (limited by fuel). LRO consists of 6 instruments and a technology demonstration. The spacecraft can be considered to have five distinct thermal systems: Instrument Module, Avionics Module (with embedded heat pipe network), Propulsion Module (within the Spacecraft), High Gain Antenna System, and Solar Array System (see Figure 1).

\footnotetext{
${ }^{1}$ Senior Systems Engineer, Systems Engineering Services and Advanced Concepts Branch, Code 592.

${ }^{2}$ Senior Thermal Engineer, Thermal Engineering Branch, 545.

${ }^{3}$ Senior Thermal Engineer, Thermal Engineering Branch, 545.
} 

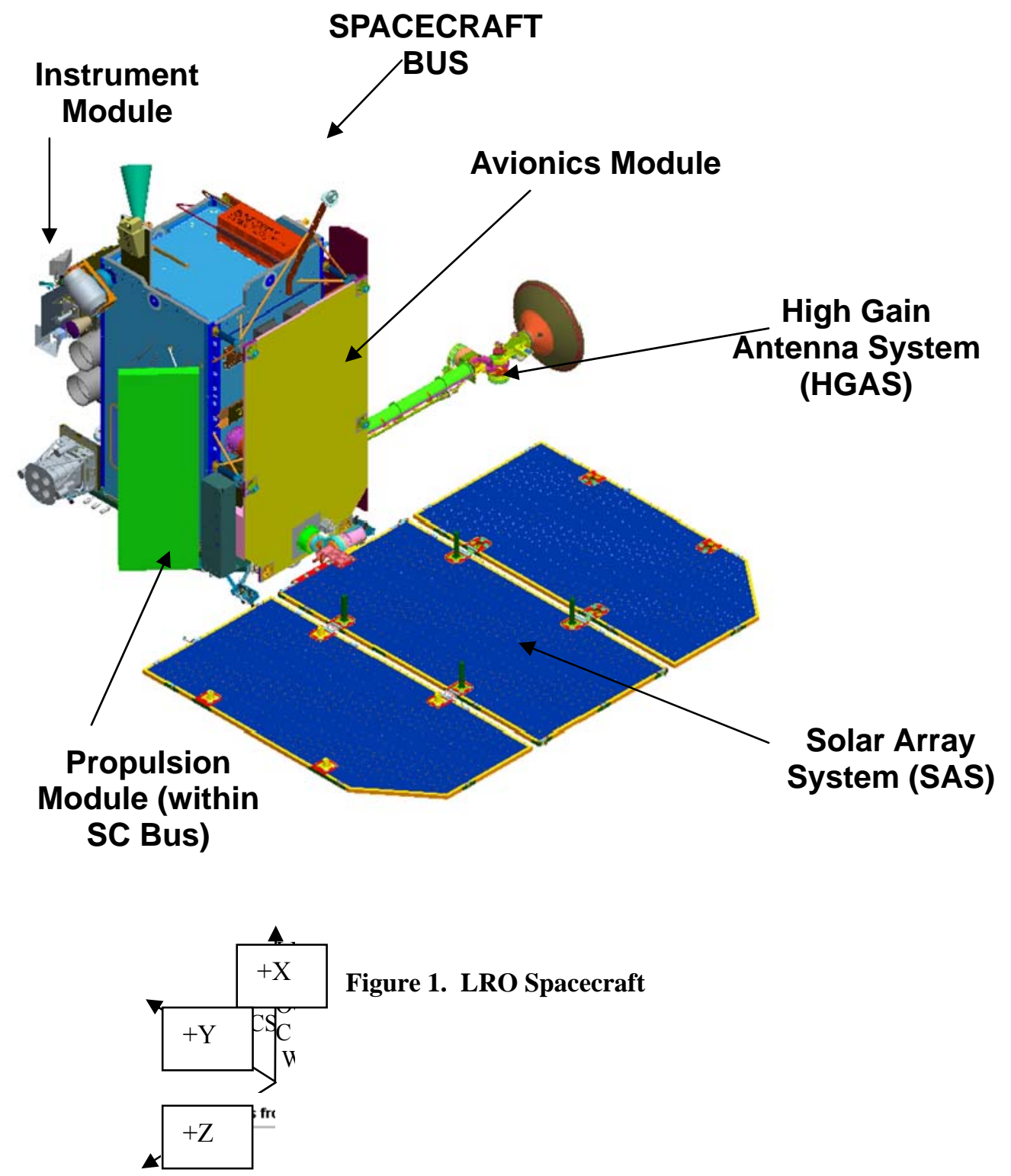

For the most part, the LRO thermal system has performed as expected under nominal conditions. However, instrument needs have resulted in unexpected thermal conditions. In the Instrument Module area, the Lunar Exploration Neutron Detector (LEND) instrument has required tighter than anticipated thermal control in order to prevent detector issues at cooler temperatures. Lunar Reconnaissance Orbiter Camera (LROC) and Lyman Alpha Mapping Project (LAMP) instruments have frequently requested unanticipated off-nadir calibration maneuvers that generally are outside of the spacecraft nominal maneuver specifications. These maneuvers are driven by science requirements not specified during the development phase of these instruments. A series of bounding off-nadir thermal analyses of the entire orbiter were performed prior to launch. These predictions are compared to actual maneuvers, including some that were considered thermally risky.

On December 21 ${ }^{\text {st }}, 2010$, LRO experienced its first severe Lunar Eclipse where the Earth shadowed the Moon for 151 minutes. As normal night periods for LRO are at most 50 minutes, this infrequent alignment of the Earth and the Moon is a driver for LRO's power system. To minimize the heater power draw while the array is shaded, 
the Spacecraft pre-heats its main avionics panel in order to minimize control heater power used during the Lunar Eclipse period.

\section{Lunar Thermal Environment}

The lunar thermal environment (modeling and environmental assumptions shown in Figure 2) presents many challenges in spacecraft design. As shown, the Moon's IR (Infrared) flux must be modeled transiently to accurately predict peak Orbiter temperatures. The heavy IR loading modeled is due to the Moon's low albedo, lack of atmosphere, and low effective regolith conduction. This is unlike most earth missions, where the IR flux is assumed to be a constant around the orbit. At high Beta Angles, the lunar IR flux is very low (as low as $5 \mathrm{~W} / \mathrm{m}^{2}$ ), whereas at low Beta Angles, the flux varies between 1335 and $5 \mathrm{~W} / \mathrm{m}^{2}$. The Sun Beta angle is defined as the angle between the vector connecting the Sun and Moon centroids and the LRO orbit plane (see Figure 3). For purposes of this paper, low Beta Angles are defined as those below Beta 30, high Beta angles as those above 70 .

The difficult thermal environment presented by the Moon required a proactive approach to thermal design as it was a significant mission driver.

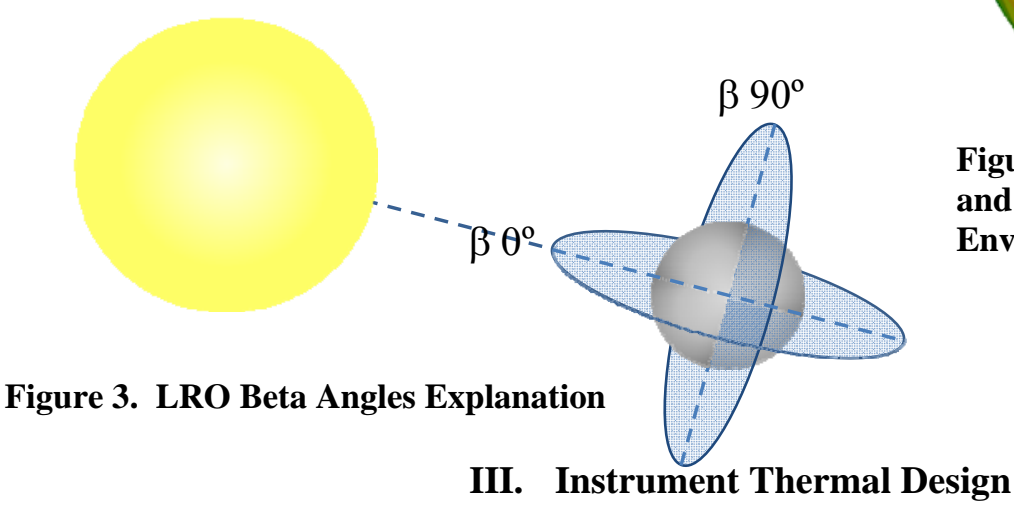

LRO has six instruments and one technology demonstration experiment. Instrument and instrument radiator orientations are shown in Figures 4 and 5. Cosmic Ray Telescope for the Effects of Radiation (CRaTER), LEND, and LAMP have Zenith (-Z) pointing radiators, which sweep through the Sun at Beta 0. The Lunar Orbiter Laser Altimeter (LOLA) has anti-sun (+Y) facing radiators (one on the electronics, one on the telescope assembly). Each LROC Narrow Angle Camera (NAC) has an angled radiator (to minimize views to the Moon) covered by a thermally isolated sunshade resulting in a steady effective sink temperature for the radiator in nominal nadir pointing. At low Beta angles, rolls and pitches of the spacecraft generally result in higher temperatures for LOLA, LROC, LEND, LAMP and CRaTER, with LROC being the most sensitive due to its low thermal mass, tight requirements, and angled radiator. Instrument calibration/off nadir pointing typically are comprised of raster scans of the Earth or star constellations, polar facing limb views at middle Beta Angles, and rolls to take profile images of significant Lunar sites. Each of these calibrations requires an examination of Sun and Moon angle qualitative assessments versus instrument radiators. Spacecraft thermal control systems have larger thermal mass and therefore are less sensitive to brief transient exposures. 


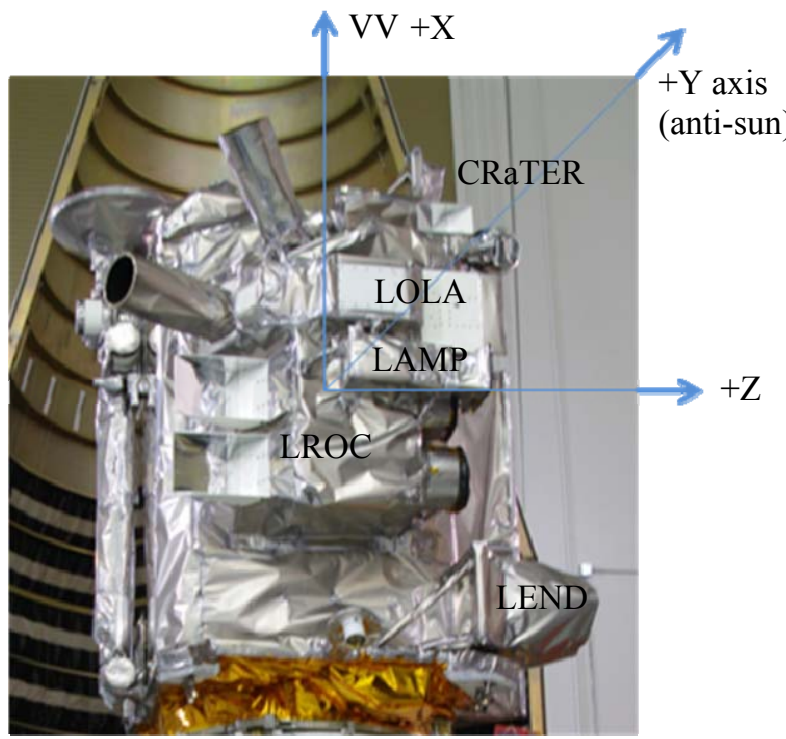

Figure 4. LRO Instrument Orientation

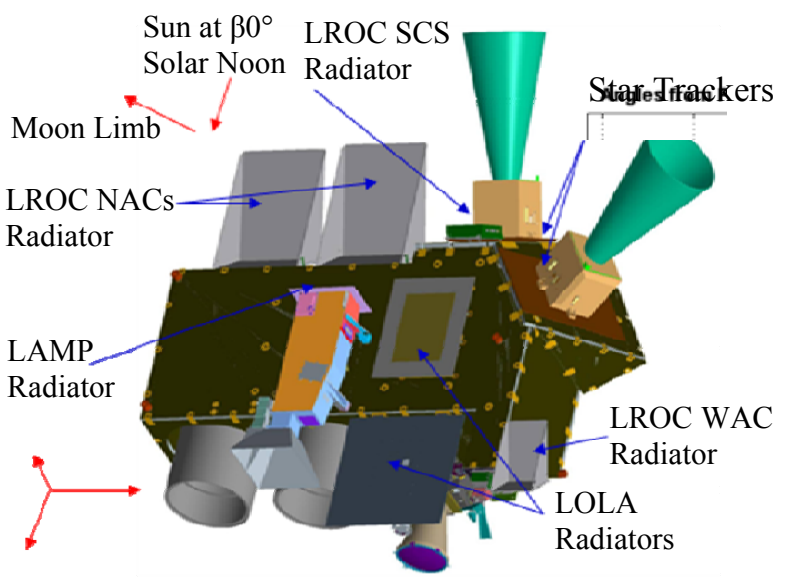

Figure 5. LRO Instrument Module Radiator Orientations

\section{Typical Off Nadir Maneuvers}

LROC is a series of 3 cameras and an electronics box. LAMP is a Lyman Alpha UV sensing instrument. The LAMP and the LROC instruments typically have two types of calibration maneuvers: star rastering, and illuminated limb views. These allow the instruments to verify pointing and calibrate out stray light effects. In addition, LAMP can perform atmospheric measurements of the Moon by pointing to the space just above the illuminated limb to

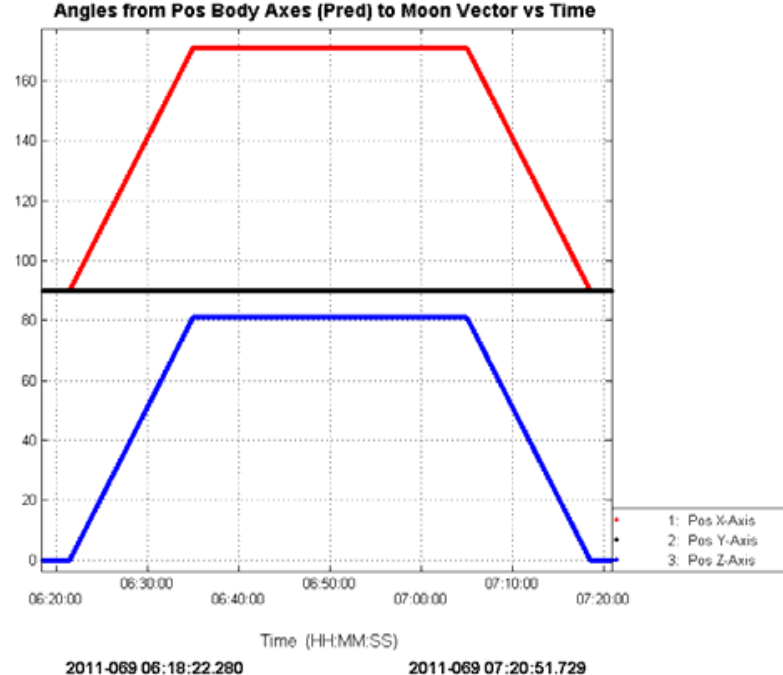

b) $+X$ normal to the Moon

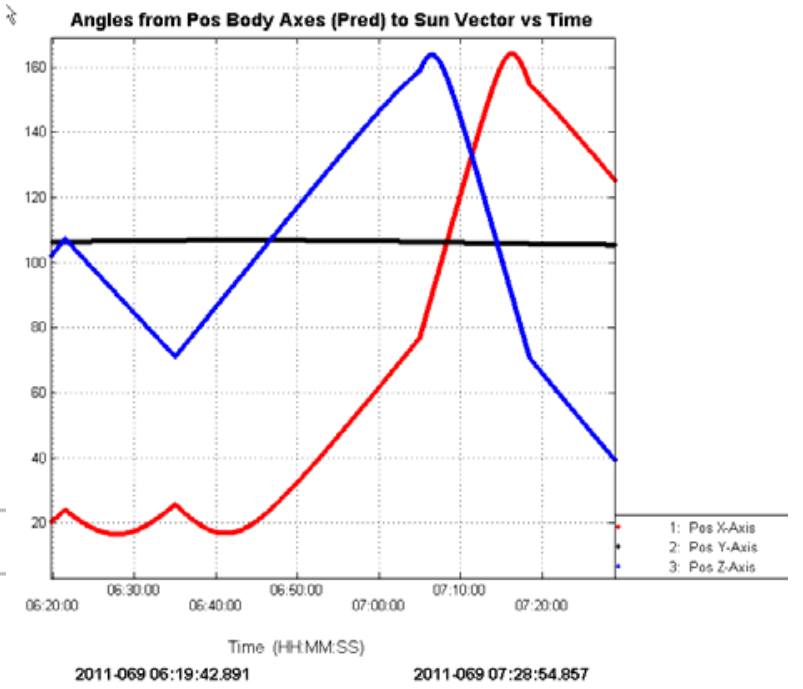

b) Sun on $+X$ face

Figure 6. Forward pitch to observe polar limb at low beta angles

measure the light spectrum reflected by the near vacuum atmosphere. The Figure 6 shows a pitch forward that looks at the polar limb at low Beta angles (the bounding worst case attitude).

Note that this pitch places the $-\mathrm{X}$ axis pointed towards the moon surface. The moon at the subsolar point approaches $120 \mathrm{C}$, but by design none of the instrument radiators are located on that face. The sun is roughly located on the $+X$ side which also is highly tolerant of sun. The following thermal case was run prior to launch to simulate a similar orbit which fixes the $+Z$ to velocity vector orbit (shown in Figure 7). Note that after 30 minutes 
the Focal Plane Assembly of LROC reaches its noise limit of $30 \mathrm{C}$. When the fluxes shown in Fig 6 were provided in flight, they were compared to Fig 7 pre-launch predict. It was decided rapidly to turn off LROC during the entire maneuver, and LAMP successfully got their limb pictures. Therefore evaluating the maneuver in Fig 6 and comparing to Fig 7 resulted in the decision to turn off LROC during the entire maneuver, and LAMP successfully got their limb pictures without damaging LROC.

Running bounding analyses prior to launch like in Fig 7 allow 1 day turn arounds for thermal safety of instruments enabling rapid operations decisions. And has resulted in dozens of off nominal calibrations and unique science opportunities.

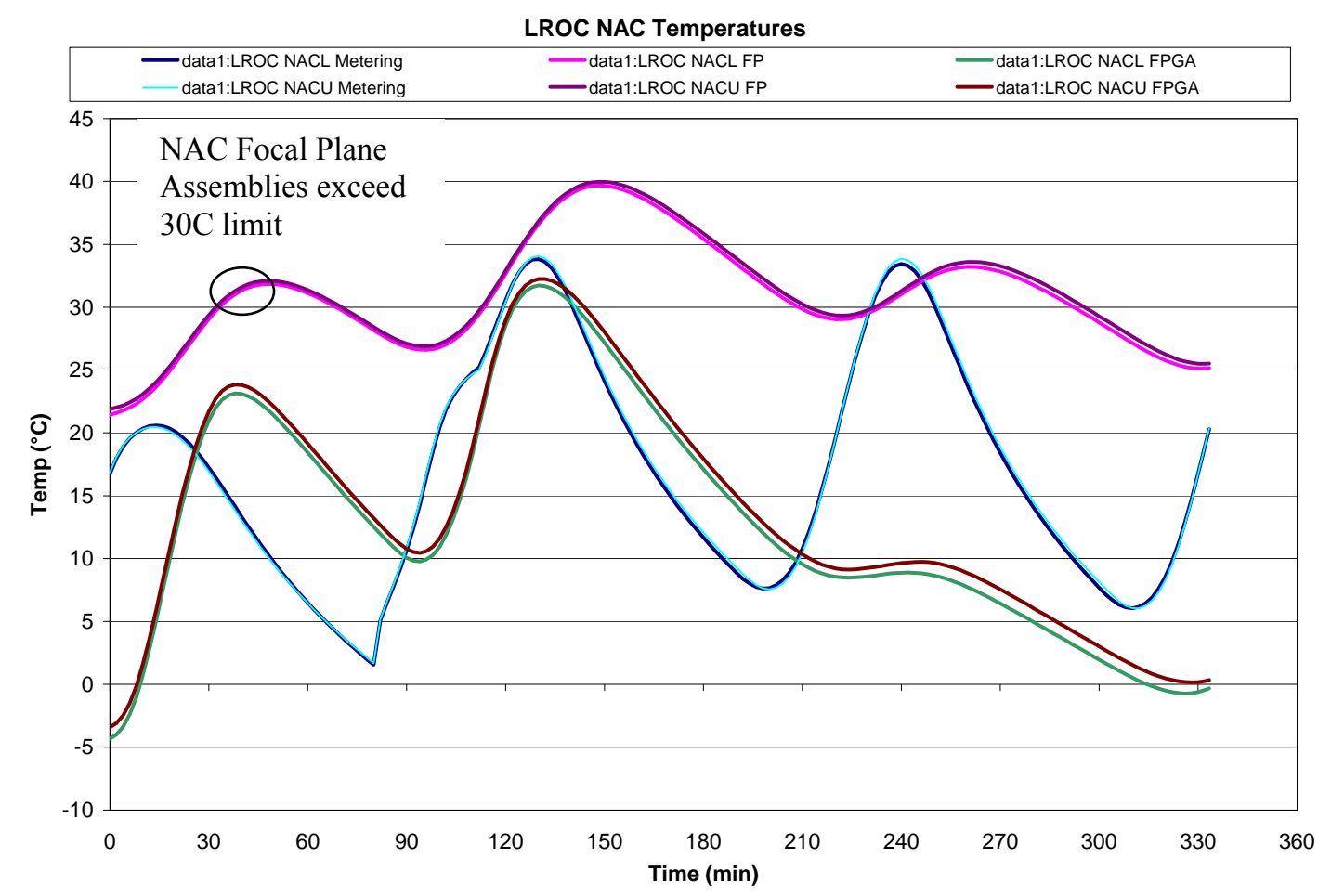

Figure 7. $+\mathrm{Z}$ to Velocity Vector Orbit Thermal Case

\section{Lunar Eclipse}

On December $21^{\text {st }}, 2010$, LRO experienced a significant Lunar Eclipse (where the Earth shadowed the moon for several hours). The LRO thermal design determines most of the energy loss from the battery during this time as non-essential flight systems are shut down and essential heater power is used to maintain the temperatures. In order to minimize the essential heater power used, the LRO spacecraft was pre-heated.

LRO was designed for significant lunar eclipses (when the Earth's shadow is cast onto the moon). In order to minimize the depth of discharge on the battery, LRO undertakes precautions to safe the instruments and preheat the avionics in order to use excess electrical power prior to the eclipse and store some of the heater power in the thermal mass of the spacecraft. Figure 8 shows the Solar Intensity versus time of the Lunar Eclipse. 


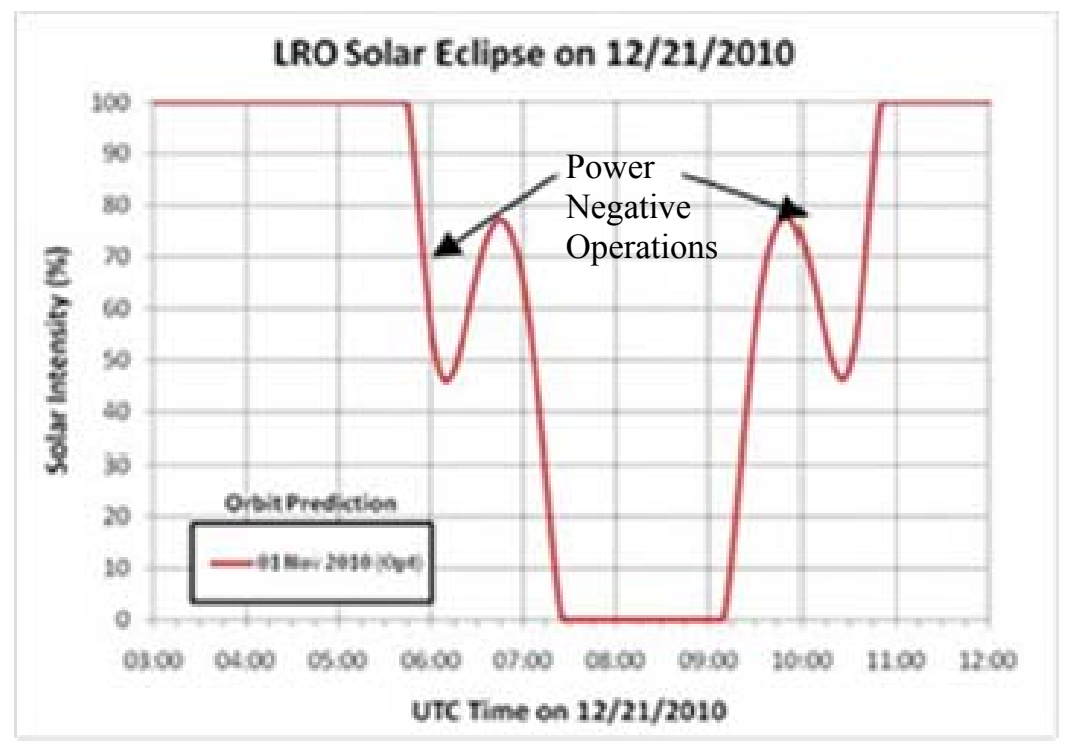

Figure 8. Solar Intensity during the Lunar Eclipse

Figure 9 shows the pre-heat and cooldown phases during the actual lunar eclipse. Note the avionics reach the maximum temperature within 3.5 hours (Battery is a separate control system and is the flat line). This results in none of the major large avionics heaters being required during the lunar eclipse (where they might otherwise consume $100 \mathrm{~W}$ ).

LRO On Orbit Thermal Data 2010/355
Lunar Eclipse Dec 21, 2010

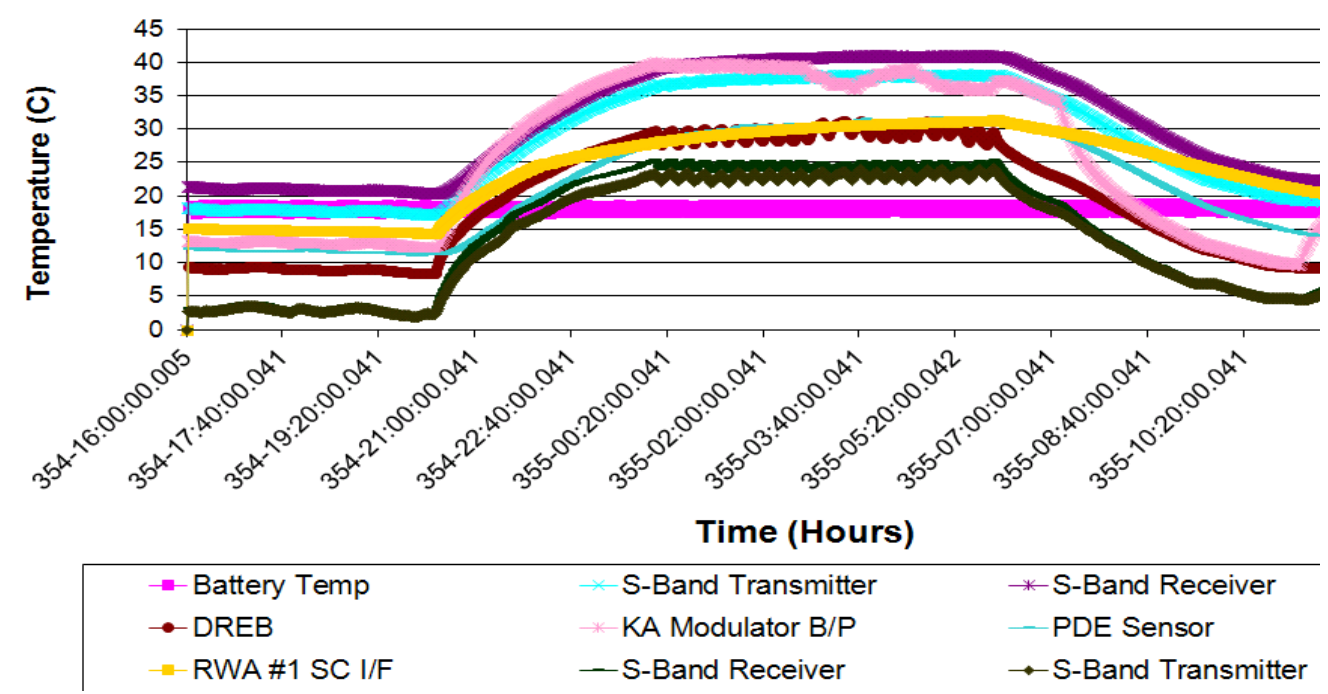

Figure 9. Lunar Eclipse LRO Pre-Heat and Cooldown

The battery Depth of Discharge (1-State Of Charge (SOC)) is highlighted in Figure 10 which shows that LRO had more than $100 \%$ margin during the lunar eclipse versus the $70 \%$ Depth of Discharge that the system was designed for. The battery is typically operated not fully recharging it to $100 \%$ to extend its life, which is why the actual curve does not recharge to $100 \%$ at the end. 


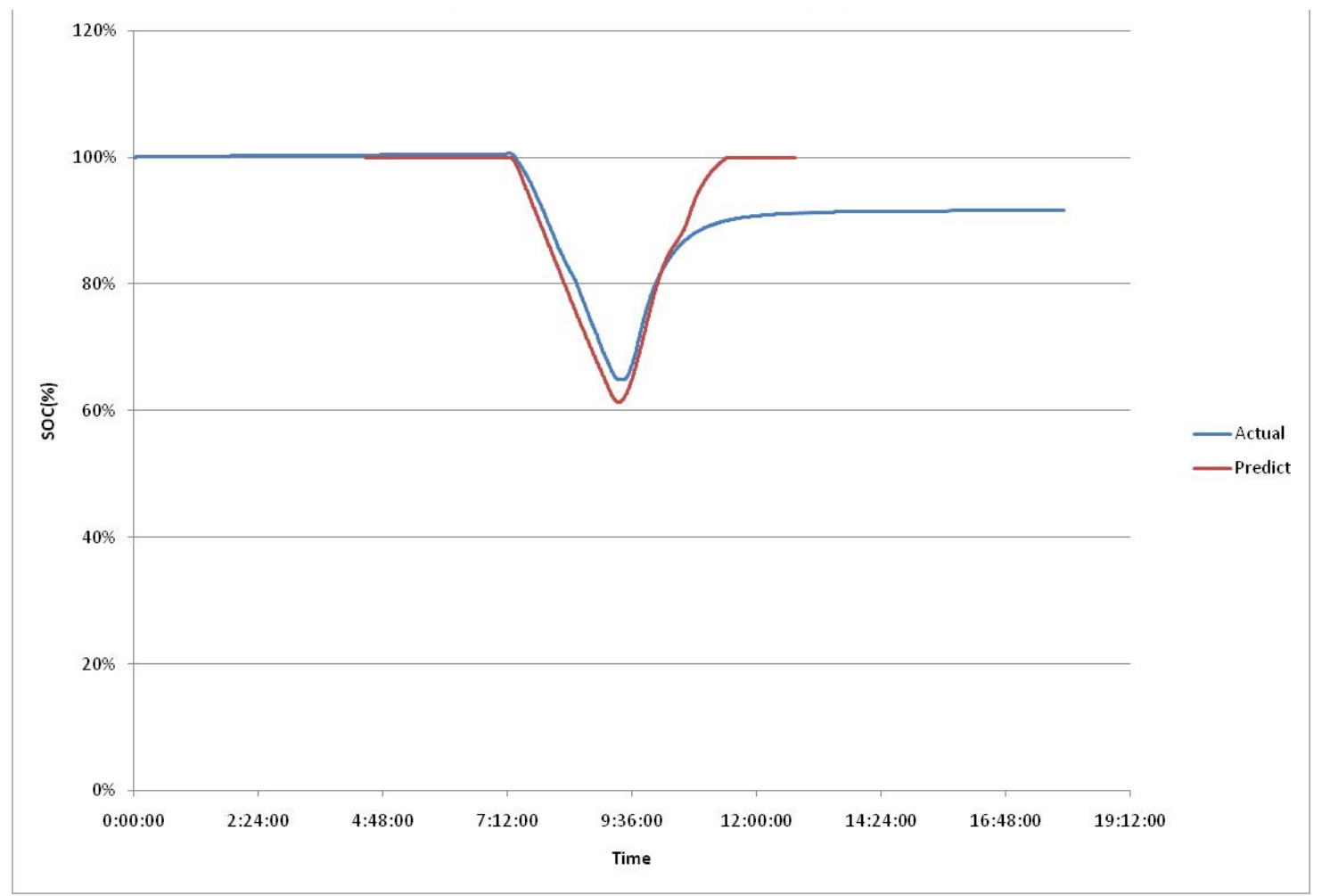

Figure 10. Battery State of Charge, Predicted vs. Actual

The Beta angles where the lunar eclipses will occur was set by the launch date and happen to be in full sun (Beta 81 degree orbits.) This was fortunate in that the maximum eclipse time was dropped by about 20 minutes versus pre-launch predicted worst-case orbits that included ordinary orbital eclipses.

\section{In Flight versus Predicted Performance of LRO}

To date, the performance of the spacecraft and instruments has remained within operational design limits and has been able to accommodate several unanticipated off-nadir calibration maneuvers. Flight data for DOY 2010-273 ( $\beta$ $0^{\circ}$ hot) and DOY $2010-361\left(\beta 90^{\circ}\right.$ cold) was compared against pre-flight bounding analytical predictions that encompassed stacked worst case conditions. In general, the performance of the instrument suite is within the analytical bounding cases for most critical components. A few notable exceptions are LEND, LOLA PCA (or Electronics) Sidewall, and Diviner/Diviner Remote Electronics Box (DREB), Figure 11. 


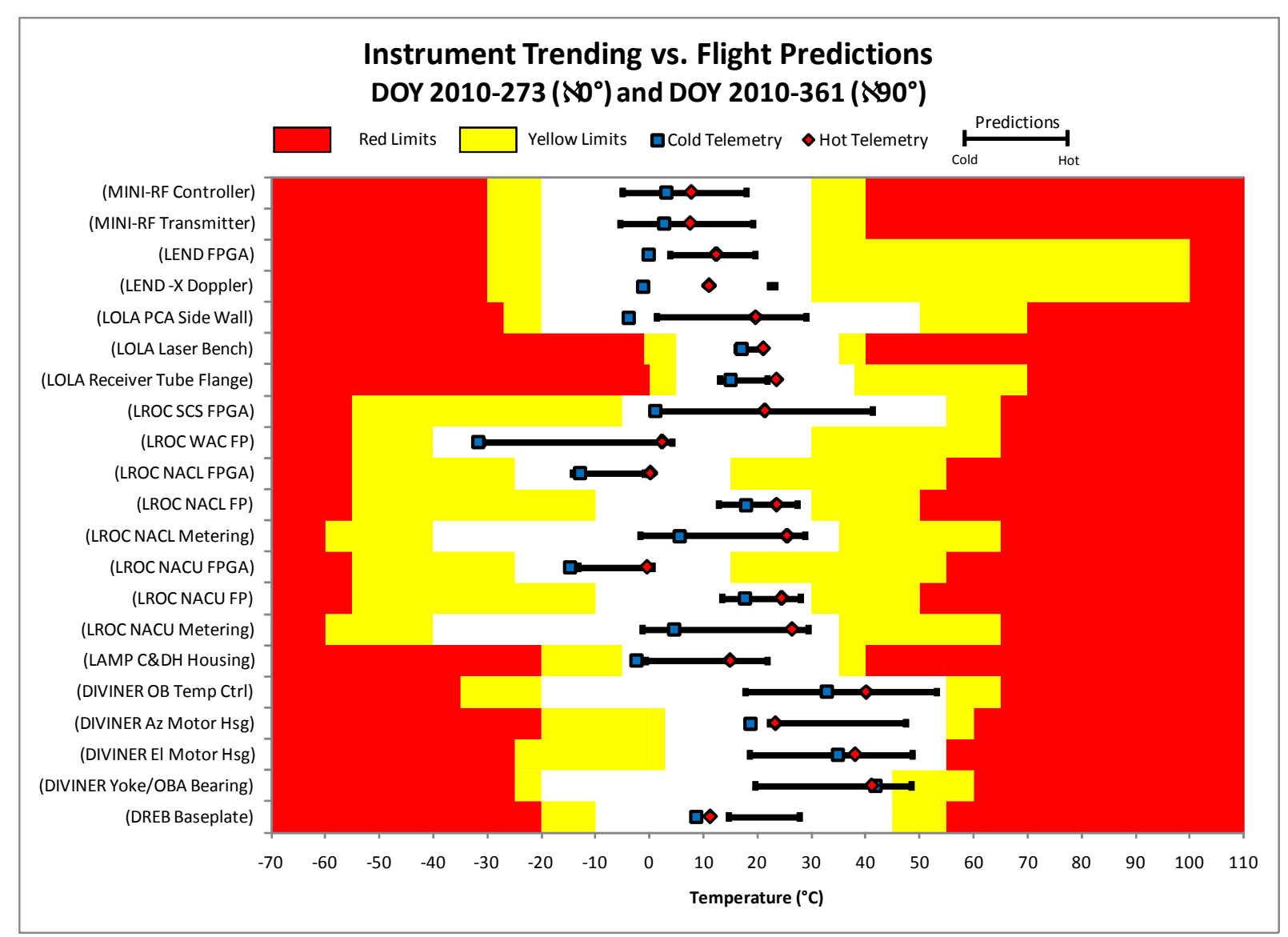

Figure 11. LRO Instrument Flight Performance vs. Predictions

LEND's performance temperatures are lower (in both hot and cold beta angles) than predicted. LEND requested that they be set at colder temperatures in the cold case and the thermal analysis (and heater predicts) were conservatively set at a warmer temperature to bound in-flight variations.

The LOLA PCA Sidewall telemetry point is the only LOLA component that shows flight performance data outside of reasonable model uncertainty compared to the analytical predictions. The overall delta between the hot and cold case temperatures (flight and predictions) remains approximately the same $\left(27^{\circ} \mathrm{C}\right.$ for predictions, $24^{\circ} \mathrm{C}$ for flight performance), it is believed that this difference could be due to a difference in power draw by the PCA between the flight article and the analytical model.

While still performing within design limits, the DREB Baseplate is showing marked differences when compared to the bounding analytical predictions. This is indicative of the relatively low degradation in the Optical Solar Reflector (OSR) avionics radiator on which the DREB box sits coupled by a network of heat pipes.

Table 1 shows the seasonal trending and beta angle trending for 2010 for the instrument suite. A small increase in temperature is seen for the trending from spring to winter seasons (Column 3 and 4), again with all components remaining within operational designs. The beta angle trending shows a decrease in operational temperatures with an increase in beta angles (Column 4 and 5), allowing some measure of freedom in determination of future orbiter maneuvers based on beta angle (e.g. orbit raising maneuvers). 
Table 1. Seasonal and Beta Angle Trending

\begin{tabular}{|c|c|c|c|c|}
\hline \multirow{5}{*}{ Instrument } & \multirow{5}{*}{ Component } & DOY 088 & DOY 273 & DOY 361 \\
\hline & & Beta $0^{\circ}$ & Beta $0^{\circ}$ & Beta $90^{\circ}$ \\
\hline & & Spring & Winter & Winter \\
\hline & & $03 / 29 / 2010$ & $09 / 30 / 2010$ & $12 / 27 / 2010$ \\
\hline & & $22: 40$ & 09:00 & $18: 55$ \\
\hline \multirow{2}{*}{ MINI-RF } & Controller & 2.5 & 7.8 & 3.2 \\
\hline & Transmitter & 2.1 & 7.5 & 2.8 \\
\hline \multirow{2}{*}{ LEND } & FPGA & 8.9 & 12.4 & -0.2 \\
\hline & Doppler Detector, $-\mathrm{X}$ & 9.3 & 11.1 & -1.1 \\
\hline \multirow{3}{*}{ LOLA } & PCA Side Wall & 16.0 & 19.7 & -3.9 \\
\hline & Laser Bench & 17.8 & 21.1 & 17.1 \\
\hline & Receiver Tube Flange & 21.3 & 23.5 & 15.0 \\
\hline \multirow{8}{*}{ LROC } & SCS FPGA & 20.1 & 21.4 & 1.1 \\
\hline & WAC Focal Plane & -5.4 & 2.4 & -31.6 \\
\hline & NACL FPGA & 0.1 & 0.2 & -12.9 \\
\hline & NACL Focal Plane & 24.2 & 23.6 & 17.8 \\
\hline & NACL Metering Structure & 20.8 & 25.1 & 6.2 \\
\hline & NACU FPGA & -1.1 & -0.4 & -14.7 \\
\hline & NACU Focal Plane & 24.6 & 24.5 & 17.6 \\
\hline & NACU Metering Structure & 21.6 & 26.2 & 4.8 \\
\hline \multirow{5}{*}{ DIVINER } & C\&DH Housing & 14.3 & 15.0 & -2.4 \\
\hline & Optical Bench Temp Cntrl Pt & 37.1 & 40.1 & 32.9 \\
\hline & Az Actuator (motor stage hsg) & 21.0 & 23.3 & 18.6 \\
\hline & El Actuator (motor stage hsg) & 36.5 & 38.1 & 34.9 \\
\hline & Yoke/OBA Bearing, A side & 38.0 & 41.2 & 41.9 \\
\hline
\end{tabular}

\section{Conclusions}

The LRO spacecraft and instruments have been performing well almost two years into the mission, although the complex thermal environment and ongoing instrument off nadir calibrations have exercised the LRO thermal design. LRO has demonstrated its ability to survive severe Lunar eclipses and perform off-nadir pointing as required by the instrument teams. Though performance has not matched predictions, the temperatures have been safely inside the operational temperatures for all of the instruments and off nadir performance has been successfully executed. Performing off nadir bounding thermal cases proved to be a good predicter of real on-orbit instrument calibrations. Operations through the two year mark have been successful despite the particularly harsh lunar thermal environment.

\section{REFERENCES}

"Lunar Reconnaissance Orbiter (LRO) Thermal On-Orbit Performance", AIAA-2010-6000 ICES 2010, Baker, C.

“Lunar Reconnaissance Orbiter Mission”, Space Science Reviews, Volume 150, Issues 1-4, 2010, Vondrak, R.R. Editor. 
"The Calibration and Early Lunar Flux Observations from the Lunar Reconnaissance Orbiter (LRO) Calorimeter", AIAA-2010-6001 ICES 2010, Cottingham, C. 


\section{ACRONYM LIST}

$\begin{array}{ll}\text { CRaTER } & \text { Cosmic Ray Telescope for the Effects of Radiation } \\ \text { DOY } & \text { Day Of Year } \\ \text { DREB } & \text { Diviner Remote Electronics Box } \\ \text { HGAS } & \text { High Gain Antenna System } \\ \text { IR } & \text { Infrared } \\ \text { LAMP } & \text { Lyman Alpha Mapping Project } \\ \text { LEND } & \text { Lunar Exploration Neutron Detector } \\ \text { LOLA } & \text { Lunar Orbiter Laser Altimeter } \\ \text { LRO } & \text { Lunar Reconnaissance Orbiter } \\ \text { LROC } & \text { Lunar Reconnaissance Orbiter Camera } \\ \text { NAC } & \text { Narrow Angle Camera } \\ \text { SAS } & \text { Solar Array System }\end{array}$

